\title{
La historia profunda de la palabra kachi: contribución a la etnohistoria protoquechua
}

\author{
The deep history of the word kachi: contribution to \\ the protoquechua ethnohistory
}

Erik Cajavilca Veramendi ${ }^{1}$

\begin{abstract}
DOI: https://doi.org/10.26512/rbla.v11i1.24954
Recebido em maio/2019 aceito em junho/2019.
\end{abstract}

\section{Resumen}

Una historia de relaciones andino-amazónicas dentro del territorio peruano subyace a la palabra quechua para sal, kachi. El estudio comparativo reveló un origen foráneo de la palabra e implicando, por lo tanto, un antiguo contacto andino-arawak en el piedemonte andino peruano que influyó en la formación de la palabra, así como en el léxico de otras lenguas de la región. En efecto, kachi se derivaría de la composición de dos protoraíces arawak, */ka?/ +*/tsi /, que denotan nombres y verbos relacionados a la práctica de la fermentación o coagulación de líquidos, una técnica muy extendida en la Amazonía para producir licores, bebidas, condimentos, entre otras cosas.

Palabras clave: Sal. Cuajar. Quechua. Arawak. Amazonía.

\begin{abstract}
A history of Andean-Amazonian relations within Peruvian territory underlies the Quechua word for salt, kachi. The comparative study revealed a foreign origin of the word and implying, therefore, an ancient Andean-Arawak contact in the Peruvian Andean piedmont that influenced the formation of the word, as well as in the lexicon of other languages of the region. Indeed, kachi would be derived from the composition of two proto-roots Arawak, $* / \mathrm{ka} / /+* / \mathrm{tsi} /$, which denote names and verbs related to the practice of fermentation or coagulation of liquids, a technique widely used in the Amazon to produce beer, drinks, condiments, among other things.
\end{abstract}

Keywords: Salt. Curdle. Quechua. Arawak. Amazon.

\section{Introducción}

Una cuestión acuciante es por qué la palabra quechua para sal, kachi, no puede ser derivada plenamente mediante el análisis intralingüístico. En ese sentido, el análisis requiere ir más allá de los límites intralingüísticos y ver si existe algún indicio que descifré la historia profunda de la palabra quechua.

1 Erik Cajavilca Veramendi Universidad Nacional Mayor de San Marcos. E-mail: 12030207@unmsm.edu.pe 
Hasta donde se sabe, el homeland quechua se localiza dentro de territorio peruano, específicamente en la sierra central peruana ${ }^{2}$ (Cerrón-Palomino 2010; Adelaar 2010), es decir, muy cerca de la selva central ${ }^{3}$ donde los antiguos arawak establecieron sus dominios. ¿Qué implicancias tiene el hecho de que los arawak se expandieron hacia el piedemonte andino en tiempos prehistóricos? En primer lugar, la mayoría de los depósitos de sal se concentran en el lado occidental del continente sudamericano ${ }^{4}$, territorio que fue el objetivo de la expansión arawak hacia el oeste y de la expansión tupí en épocas posteriores (Hornborg y Eriksen 2011:137), asimismo el territorio coincide con la mayor concentración de lenguas aisladas al oeste del continente, por encima de los $20^{\circ}$ de latitud sur (Dahl et al. 2011:217).

Por lo tanto, la expansión arawak - así como otros grupos - hacia occidente estuvo económicamente motivada con el fin de abrir nuevas rutas comerciales, pero no fue una colonización opresiva, sino todo lo contrario. Los protoarawak son originarios del interior de Brasil ${ }^{5}$, donde establecieron relaciones con otros grupos importantes de la Amazonía. En ese sentido, creemos que la colonización arawak de los Andes peruanos y las tierras bajas adyacentes tuvo una importante consecuencia tecnológica, cultural y lingüística en la etnogénesis de antiguos pueblos del territorio peruano. Así, la palabra kachi oculta, en su historia profunda, pistas acerca de la integración comercial andino-arawak de tiempos prehistóricos. Su formación léxica sigue un patrón que se repite en otras lenguas amerindias. Este patrón tiene que ver con la tecnología y el modo de preparación o refinamiento de la sal, es decir, ya sea la sal extraída de agua del mar, río, marismas, de la tierra, de la roca ${ }^{6}$ o de las cenizas vegetales, todas deben ser hervidas en disolución acuosa y esperar que se asiente el coagulado, con excepción de la sal que se forma naturalmente por evaporación en lugares calurosos y secos. De hecho, el cocimiento es una práctica muy conspicua para la materialización de los productos amazónicos más representativos como los venenos, las infusiones medicinales, las bebidas fermentadas, entre otros.

Utilizando el método comparativo interlingüístico y el análisis de cognados intentaremos esclarecer el origen de la palabra kachi, apoyándonos también en otras raíces léxicas que siguen un desarrollo análogo. En ese sentido, la empresa de este documento tiene doble mérito, por un lado, aportar a la etimología de la palabra kachi y, por otro lado, aportar a las teorías de etnogénesis quechua-andina.

\footnotetext{
${ }^{2}$ Esta zona abarca la sierra de los departamentos de Ancash, Huánuco, Pasco y Junín.

${ }^{3}$ Abarca territorios de los departamentos de Huánuco, Pasco, Junín y Ucayali. Esta zona ha sido conocida como el territorio de los campa (arawak), donde se localiza también el Gran Pajonal.

${ }^{4}$ Véase, por ejemplo, las láminas al final del libro de Wörrle (1999) que retratan los yacimientos de sal en Sudamérica.

${ }^{5}$ Estamos de acuerdo con el homeland arawak sugerido por Aikhenvald (2015:387), en el territorio situado entre los ríos Negro y Orinoco.

${ }^{6}$ Nos preguntamos si fueron los protoarawak subandinos los primeros que cocieron la sal de roca para ablandarla.
} 
Empezaremos describiendo el contexto de la distribución de las fuentes de sal en el territorio andino, especialmente en el Perú, para entender mejor el movimiento y comportamiento de los pueblos amazónicos.

\section{Los yacimientos de sal reportados en las crónicas coloniales}

Muchos de los depósitos de sal reportados en la Colonia fueron explotados por los europeos después de la conquista; en ese sentido, si bien los yacimientos de sal podrían haberse conocido desde mucho antes de la conquista, poco sabemos de los grupos nativos que las explotaban y en qué magnitud.Según se estima, en la época del Tahuantinsuyo, se utilizaban grandes cantidades de sal para la conservación del pescado. Este alimento conservado era consumido desde la costa hasta la sierra por personas de todas las clases sociales (Buse 1975, 2:568). En las sociedades asentadas en tierras ricas de sal $(\mathrm{NaCl})$, la conservación por salado debió ser muy común; en caso contrario, en territorios donde la sal era escasa, se debieron utilizar otros métodos alternativos de conservación; por ejemplo, en la Amazonía, los nativos emplean el ahumado y el secado. Así también, los incas gastaban grandes cantidades de sal para quemar la tierra de los infractores y volverla infértil, incluso arrojaban sal en los santuarios paganos o ídolos locales (Jiménez 1879:283-84). Según Arriaga (1621:37) las mujeres incas quemaban sal y arrojaban las cenizas al aire para deshacerse de la niebla ${ }^{7}$. Los cuerpos de los parientes fallecidos eran conservados con sal y se los colocaban en algún lugar de la vivienda, práctica milenaria que data desde hace miles de años. El más temprano indicio es encontrado en Chinchorro, en la costa norte chilena y también en La Paloma, hace 6000 años, en la costa central peruana (Moseley 2001:101,111). Se deduce, por lo tanto, que la visión original del moderno mallqui inca tenía que nacer en zonas con abundantes recursos de sal, en sentido opuesto, en las tierras lluviosas tropicales no es usual encontrar prácticas de momificación o conservación de cadáveres.

\section{Los yacimientos de sal en el mundo andino}

En el mundo andino, la sal podía ser explotada desde diversas fuentes localizadas tanto en la costa del Pacífico como en las tierras altas. Las fuentes de sal estaban en los litorales, en el agua de mar o de río, en las vertientes, entre otras (Wörrle 1999:29-44). Patiño (1992:24-37) clasifica en dos grupos los yacimientos de sal de los pueblos tradicionales del occidente de Sudamérica: la sal común (marina, terrestre y de manantial) y sustitutos de la sal (minerales y vegetales).

La abundancia de yacimientos de sal en los países andinos siempre fue motivo de admiración por los europeos. Cobo no deja de admirar la abundancia de yacimientos de sal en los llanos costeros del Pacífico y dice que hasta en los desiertos secos hay tanta sal que podría proveer a todo el planeta (Cobo 1890:183). Esto coincide con el intenso comercio de pescado salado, por lo que se infiere una

\footnotetext{
${ }^{7}$ Una descripción sintetizada del aspecto mágico-religioso de la sal en el antiguo Perú puede leerse en Buse (1975, 1:741-44).
} 
intensa explotación en las salinas marinas. Con mayor razón, una vez conquistada el Chinchaysuyu, en el siglo XV (D’Altroy 2015:97-98), se beneficiaron las salinas costeras para alimentar al creciente imperio con comida conservada en sal. Siempre se mencionan ricos yacimientos salíferos no explotados en la época de la conquista, por lo tanto, al parecer la falta de sal no era un problema para el Tahuantinsuyo.

A partir de los archivos coloniales (s. XVI), tenemos conocimiento de las salinas de sal colorada en Santa, al norte de la costa peruana, que abastecía a los pueblos andinos del callejón de Huaylas y Conchucos (Jiménez 1881:CXL). La salina de Huaura, ubicada a $150 \mathrm{~km}$ de Lima, por la costa, que provee sal de buena calidad y tiene fama de ser la más grande del Perú (ibíd.: CXLIIICXLIV). Asimismo, las salinas de Paita en Piura, admirada por los cronistas y de la que se extraen rocas enteras de buena sal (Jiménez 1885:229). Algunos pueblos del interior que carecían de yacimientos importantes, tales como Loja en Ecuador, se abastecían de sal de estas salinas piuranas (Jiménez 1897:200, 209).

En la sierra, se describe, a $28 \mathrm{~km}$ hacia el oeste de Guamanga (Ayacucho), una enorme mina de sal colorada con manantiales interiores de agua salada del que los indígenas extraen las rocas de sal cristalina o bien sal blanca cuajada a partir del agua salada secada en pozos (Jiménez 1881:126-27). Más lejos, $45 \mathrm{~km}$ hacia el sur, hay un manantial de agua salada de la que se extrae sal por cocción de la salmuera y, de la misma manera, a casi la misma distancia hacia el norte, cercano al río Xauxa, se explotan otros vertederos de agua salada, pero en menor escala (ibíd.:128). A $33 \mathrm{~km}$ del pueblo de Villa Rica de Oropesa (Huancavelica) se extraía sal cociendo el agua de dos manantiales de agua salada (Jiménez 1885:8). Los indígenas de Arequipa sebeneficiaban de la sal de roca turbia extraída de minas cerca de Lluta, mientras que en Guanbo (Huambo), en la misma región, se obtenía sal cuajada por filtración (ibíd.:47). En la capital de los incas, antes y ahora, se obtiene sal de excelente calidad en San Sebastián, muy cerca de la ciudad, y la de Maras (ibíd.:196). Más al sur, en el altiplano boliviano, hay un gran yacimiento a $8 \mathrm{~km}$ de Caquingora (Pacajes) 1lamado Yaribaya ${ }^{8}$ cuya sal se cuaja fácilmente con el frío (ibíd.:62). Había una salina lacustre entre los pueblos de Azángaro y Chupa en Puno, y del cual los lugareños extraían y vendían sal de diferente color. No les bastaba a los comensales la sal producida del lago, sino que también compraban una arcilla medio blanca llamada cchaco ingerida como condimento (Raimondi 1874:212).

La isla de Puná, provincia de Guayas en Ecuador, es conocida por sus salinas costeras que abastecían de mucha sal a los pueblos del interior, entre ellos a las ciudades andinas de Quito por el norte (Jiménez 1897:13) y a Cuenca por el este (ibíd.:161). Fue menester para el quiteño Atahualpa conquistar la isla debido al bloqueo impuesto por sus dueños, leales a Huascar, y porque no había otro yacimiento disponible en los alrededores (Herrera 1728:375-76). Cuenta Juan de Castellanos (s. XVI) que, en Zipaquirá y Nemocón, al norte de Bogotá, se obtenían bloques de la sal más blanca y sabrosa de toda la región, para lo cual se cocía

\footnotetext{
${ }^{8}$ Probablemente se refiera al salar de Jayuma Llallagua del distrito de Coro Coro.
} 
la salmuera en vasos de cerámica (Castellanos 1886:95). Para el cronista español Vázquez de Espinosa (s. XVII), las salinas venezolanas de Araya, cerca a Cumaná, «son las más copiosas y abundantes de sal que tiene el universo» (1948:50).

\section{Los yacimientos de sal al este de los Andes}

En la Amazonía, el cloruro de sodio como tal es en realidad escaso. Los mayores recursos de sal estaban, por un lado, al pie de las estribaciones andinas, en su lado oriental, y, por otro lado, en las lejanas costas atlánticas brasileñas. En algunos casos, la sal de las vertientes aflora en forma de roca en los cerros o es arrastrada por la lluvia formando manantiales o ríos salados ${ }^{9}$ que los indígenas evaporan en recipientes para que condense la solución salina.

En la selva central peruana, el depósito de sal más famoso es el de Cerro de la Sal. Las vetas de sal de roca fueron descubiertas en 1635 por fray Jerónimo Jiménez a orillas de un afluente del Paucartambo, distrito de Villa Rica en Pasco y tendría una extensión de $33 \mathrm{~km}$ y un ancho de veta de $25 \mathrm{~m}$. El beneficio de la sal gema se remontaría mucho antes de su descubrimiento, en donde acudían nativos de diversos pueblos lejanos (Raimondi 1876:192-93). Esta sal gema bruta del Cerro de la Sal que los nativos campa cuecen ${ }^{10}$ en agua, está mezclada con tierra y es bastante cáustica debido, tal vez, a la presencia de sulfatos en su composición (Renard-Casevitz 1994:84, 88); esto concuerda con la descripción de la sal gema hecha por el ingeniero Lino (1962:40), la cual contiene cierta cantidad de arcilla ferruginosa y sulfato cálcico.

Por otro lado, hay también depósitos de sal gema en el río Huallaga como el de Callanayaco, antes de llegar al Pongo de Aguirre, en San Martín. Dice Raimondi que este prolífico yacimiento era visitado por nativos de diversas etnias (Raimondi1874:397). Asimismo, los cerros de sal de Pilluana en Picota, margen derecho del Huallaga en San Martín, de donde se extraen vetas hasta de tres colores distintos de sal. Estos cerros de sal tienen una extensión de $1.5 \mathrm{~km}$ (Raimondi 1876:423-24). En el río Ucayali, fueron los pano quienes dominaron el comercio de sal, como los shipibos quienes extraían sal del río Pisqui, afluente del Ucayali (Izaguirre 1926:443); mientras que los cashibos hacían lo propio de un cerro de sal en el Aguaytía (Tessmann [1930] 1999:77). Los secoya ecuatorianos del río Wajoyá ${ }^{11}$ emprendían un arduo viaje hasta el centro de Loreto, Perú, para conseguir sal; según el informante, los secoya extraían bloques de sal de algún yacimiento

\footnotetext{
${ }^{9}$ Por ejemplo, Potiaríni es un río de agua salada, tributario del Perené en su curso superior y más abajo está Tivíha, un manantial salado en el lado derecho del río Tambo (Weiss 1975:314, 555).

${ }^{10}$ La cocción de la sal de roca permite el ablandamiento o eliminación de iones de calcio o hierro.

${ }^{11}$ Es un río afluente del Aguarico, a su vez, afluente del Napo, en la provincia de Orellana, Ecuador.
} 
del Marañón ${ }^{12}$ a cinco días a pie de Iquitos (Cipolletti y Payaguaje 2008:83-84). Según Karsten ([1935] 2000:108), los jívaros del Ecuador conseguían sal al cocer el agua salada de los ríos; estas fuentes estaban, por un lado, en la confluencia del río Mayaiku ${ }^{13}$ con el Zamora en la provincia de Zamora Chinchipe, o también, en la confluencia del río Curi-Curi con el Mangosiza en la provincia de Morona Santiago, de este también se destinaba a la venta en Macas. Por otro lado, los jívaros del Perú extraían sal de minas ubicadas en el río Nieva, provincia de Condorcanqui, y en el río Apaga, provincia de Alto Amazonas (Tessmann [1930] 1999:196); ambos ríos pertenecen a la cuenca del Marañón. Cerca de allí, en Alto Amazonas, los chayahuitas extraían sal del río Cachiyacu, afluente del Huallaga (ibíd.:215). En síntesis, la amplia zona entre el Huallaga y el Ucayali forma un enorme depósito de tierras salíferas mezclada con areniscas multicolores como describe Poeppig (1836:336).

Ahora bien, en territorio Brasileño, los mayores depósitos de sal estaban en los estados costeros del Atlántico, desde el estado de Pará hasta el estado de Río de Janeiro, que actualmente son explotados por los brasileños (Diniz et al. 2015), siendo la zona conocida como Costa Semiárida Brasileña — desde el estado de Marañón hasta Río Grande del Norte- la más prolífica del país (Diniz y Vasconcelos 2016). Respecto a los informes coloniales (s. XVI-XVII) de estos mismos yacimientos, consúltese en Sousa (1879), Heriarte (1874:21-22), Hemming (1978:171).

En definitiva, todos estos lugares mencionados se distribuyen con mayor tendencia en las zonas áridas de las costas del Pacífico y del Atlántico y a lo largo del corredor andino, mientras que la descripción de yacimientos en el interior del continente es escasa, por no decir nula. Esta distribución de los yacimientos de sal en Sudamérica concuerda con el hecho de que los dos pueblos que consumen menos cloruro sodio en el mundo son del interior del Amazonas: los yanomami ${ }^{14} \mathrm{y}$ los del río Xingú (Elliott y Brown 2007).

\section{Las sales vegetales}

Las salinas de los litorales y de las vertientes andinas no fueron y no son los únicos medios para conseguir sal. También se obtenía un tipo de sal picante y amarga a partir de la cocción del caldo alcalino ${ }^{15}$ obtenida previamente por lixiviación de cenizas vegetales; sin embargo, estas sales se caracterizan por su bajo contenido de sodio y un alto contenido de iones potasio (Echeverri y RománJitdutjaaño 2011:495) a diferencia de las sales de origen mineral que contienen más

\footnotetext{
${ }^{12}$ En el texto se menciona el río Huallaga, sin embargo, es muy poco probable que demoren solo cinco días a pie en ir de Iquitos al Huallaga.

${ }^{13}$ Evidentemente, se trata del río Nangaritza.

${ }^{14}$ Los yanomamo habitan en el extremo sur de Venezuela y entre los estados brasileños de Amazonas y Roraima (Aikhenvald y Dixon 1999:342-43).

${ }^{15}$ El caldo alcalino es la solución filtrada o lixiviada de cenizas de partes vegetales (raíz, hoja, corteza). Esta solución concentrada es hervida hasta que el agua se evapore y se forme el coagulado (Echeverri y Román-Jitdutjaaño 2011:493).
} 
sodio. Por ejemplo, el padre Cobo (s. XVII) relata cómo los nativos de Venezuela hacían sal de esta manera, a partir de cogollos de palmera, y los de Popayán, en Colombia, a partir de una hierba cocida en agua (1890:237). Los indígenas de la margen izquierda del río Negro en Brasil, reportados por Heriarte (s. XVII), hacían sal de una planta que llaman capinasu ${ }^{16}$; esta sal también estaba destinada a la venta $(1874: 49,77)$. Según Acuña (s. XVII), los omaguas de los ríos Napo y Amazonas hacían sal de las cenizas de hojas de palmera (Hemming 1978:232). Humboldt cuenta que los nativos de Javita de Venezuela, en la margen izquierda del río Temi, estado de Amazonas, hacían sal de las cenizas del fruto de la palmera seje, que abunda entre Javita y el Caño Pimichin. La sal producida era una mezcla de cloruro de potasio, soda, cal y otras sales terrosas (Humboldt y Bonpland 1907:365-66).

Los vegetales preferidos para la producción de sal son las palmeras, como consta en la investigación de Echeverri (2001:408), quien utilizó 27 especies de palmeras de 60 especies vegetales en total en una comunidad uitoto de la Amazonía colombiana. No obstante, las plantas de la familia Amaranthaceae, que habita en todos los continentes, son famosas por sus cenizas ricas en iones de potasio, por ejemplo, la especie Salicornia herbacea L. del Medio Oriente (Majno 1991:50). En la Amazonía, una especie de dicha familia es la planta carurú que es muy utilizada como materia prima de la sal vegetal; por ejemplo, en tariana (AIK), iwi denota 'sal' $\mathrm{y}$ 'carurú' debido a que el condimento se obtiene a partir de dicha planta que crece en las piedras; en tuyuca (BAR), móa denota 'sal'y 'carurú' que crece en las rocas de los raudales, es interesante que la planta no solo sirve para hacer sal, sino también, para preparar medicina y barbasco. Koch-Grünberg (1913) explica la segunda acepción del término tukano moá: «nombre de una pequeña planta (p. ej. karurú) que crece en las rocas de los raudales, de cuyas cenizas los indios preparan sal». Carurú es, pues, el nombre vernacular usado por hablantes de castellano y portugués, y refiere a una especie de la familia Amaranthaceae o también Podostemacea (según identifica Koch-Grünberg 1913). Del mismo modo, Stradelli ${ }^{17}$ (1929) identifica la misma planta, caáryrú, con similar utilidad. Heriarte (1874:78) reporta cururé, la planta de la cual los indígenas del río Negro ${ }^{18}$ preparaban sal en el siglo XVII.

Se ha adjudicado un beneficio meramente culinario a la sal vegetal, pero con una calidad más baja que la sal mineral, es decir, considerada solo como «una solución emergente de carácter secundario» (Wörrle 1999:41). Sin embargo, la sal vegetal era más que un simple condimento, sino era también parte de la sabiduría herbolaria: especia, medicina y veneno. Para los huitotos la sal vegetal tiene propósitos rituales, así también, como aditivo de tabaco, fármaco y especia (Echeverri 2000:33). Las cenizas vegetales siempre fueron usadas desde tiempos remotos por su rico contenido de minerales y sales alcalinas ${ }^{19}$. La práctica de

\footnotetext{
${ }^{16}$ Es una especie de palmera.

${ }^{17}$ En el mismo diccionario, se reporta el cognado caruára 'esp. hormiga' (STR).

${ }^{18}$ En la cuenca del río Negro, existe una catarata llamada Carurú; cerca de allí Wallace probó dicha planta hervida con pescado y la describió como una especie de Podostemaceae (Wallace 1889:241, 340).
}

19 Nos referimos a sales de iones sodio, potasio, magnesio y calcio que pueden estar 
obtener cenizas vegetales con alto contenido alcalino sirvió posteriormente para crear el nombre álcali: la palabra viene del árabe al-qilì, donde al- es el artículo y qilì denota 'cenizas carbonizadas de salicor ${ }^{20}$ ', siendo cognado con el arameo qělā́ 'quemado' y el acadio qalū 'quemar' (Klein 1966:50). No obstante, el exceso de sal vegetal puede causar envenenamiento debido a sus propiedades corrosivas (Echeverri 2001: 464; Echeverri y Román-Jitdutjaaño 2011:499). Dependiendo de la mezcla, la sal vegetal puede ser más o menos venenosa, por ejemplo, una mezcla rica en carbonato de potasio se debe ingerir solo ocasionalmente o en pocas comidas, mientras que una mezcla rica en cloruro de potasio puede ser ingerida con más frecuencia ${ }^{21}$.

En suma, la producción de sal a partir de cenizas vegetales es una práctica muy antigua realizada por pueblos que no tienen acceso a salinas ${ }^{22}$ naturales $y$, especialmente, por pueblos tropicales donde la lluvia no permite la acumulación de salinas . En el caso de Sudamérica, los pueblos que acuden a esta práctica son mayormente los del interior del continente, aquellos alejados de las estribaciones andinas y de las costas atlánticas.

\section{Aspectos léxico-semánticos}

Muy poco se dice, en general, de los orígenes etimológicos de las palabras que denotan sal. Por lo que sabemos a ciencia cierta, la sal era explotada desde los tiempos neolíticos europeos (Weller 2004) y se presume que el consumo se remontaría más atrás en el tiempo. En los diccionarios etimológicos del griego (Beekes 2010) y latín (Vaan 2008), ninguna idea adicional se propone, más bien, tan solo se remite a la fuente indoeuropea con el mismo significado: «sal». Por ejemplo, en la sección ETYM, Beekes (2010:75) se limita a afirmar que el término es una "antigua palabra encontrada en la mayoría de las lenguas indoeuropeas», cuya forma hipotética es * $\mathrm{seh}_{2}-l$, pero no esclarece el origen semántico.

¿Qué podría motivar semánticamente la formación de los términos para sal? En realidad, es un trabajo arduo descifrar el origen de las palabras en el ámbito alimenticio debido a que todo lo que es natural — plantas, animales, minerales - se decanta en palabras que provienen de tiempos inmemoriales. No sucede así con las palabras que denotan objetos manufacturados o nociones abstractas, ya que estas se derivan de las primeras.

combinados o no con aniones carbonato, cloruro y sulfato (Echeverri 2001:398; Echeverri y Román-Jitdutjaaño 2011:498).

${ }^{20}$ Salicor o salsola es una especie de la familia Amaranthaceae.

${ }^{21}$ Esta afirmación la hemos deducido a partir de los datos de toxicidad y de la escala EPA (Environmental Protection Agency) tanto del cloruro de potasio como del carbonato de potasio en la página web de Pubchem: https://pubchem.ncbi.nlm.nih.gov/compound/4873 https://pubchem.ncbi.nlm.nih.gov/compound/11430

${ }^{22}$ En Echeverri \& Román-Jitdutjaaño (2011:498), se muestra una lista de lugares y países donde la gente produce sales vegetales. 
Ahora bien, existe un patrón que se repite en léxico de la sal que remite a un grupo de palabras fonéticamente similares. El problema con este grupo es que hace referencia a otros objetos naturales, por lo que se corre el riesgo de caer en la circularidad referencial. No obstante, en principio, seguiremos este patrón formasignificado para sostener la tesis de posibles contactos lingüísticos en primer lugar. Los diccionarios etimológicos de dos familias de lenguas asiáticas —altaicas y dravídicas - nos proporcionan datos interesantes respecto a la presunta etimología de la sal. Presentamos una lista de los términos similares, extraídos de los diccionarios de Starostin et al. ${ }^{23}$ (2003) y de Burrow y Emeneau (1984).

Significado: «sal», «cualidades de sabor»

- En proto manchú-tungús, *ร̌ujar-24 'amargo, ácido'; en prototúrquico, *dūr 'sal'; en protojaponés, *túrá- 'insoportable, duro, amargo'; en protocoreano, *čjarr'estar salado' (Starostin et al. 2003:398).

Significado: «sal», «insecto», «fermentado»

- En proto manchú-tungús, *saKV ‘secado (de carne, pescado)'; en protocoreano *sòkòm 'sal' (ibíd.:1204).

- En protomongol, *sag 'huevos de insectos, liendres'; en prototúrquico, *sakirtka 'garrapata'; en protojaponés, *su(n)karu 'avispa excavadora' (ibíd.).

- En proto manchú-tungús *saku 'agua estancada, agua turbia', 'volverse turbio'; en protomongol, *saga- /*saka- 'fermentar, escurrir'; en prototúrquico, *sakìr /*sagìr 'goma, resina'; en protocoreano, *sàk-/*sàk- 'exudar, efervescer, fermentar', 'pudrirse, echarse a perder', 'fermento, levadura' (ibíd.).

Significado: «sal», «hierba»

- En proto manchú-tungús *sipa 'ajo'; en protomongol *sibag 'artemisia'25; en prototúrquico *sibüt 'eneldo, cilantro'; en protojaponés *simpù-/*sìpà 'astringente, agrio', 'sal'; en protocoreano *psí- 'hígado', 'amargo' (ibíd.:1238).

Significado: «sal», «picante», «insecto», «escocer»

- Kaț 'palpitar y doler (por picadura, mordedura venenosa, pinchazo, dolor de muelas)', 'dolor', 'ser demasiado condimentado o picante', 'estar enojado o indignado', 'amargura, pungencia, veneno, astringencia'; katutt-ēru 'avispa, avispón' (tamil) ${ }^{26}$; kațppam, kațma 'pungencia, dureza, bravura, fuerza del ponche, vinagre' (malayalam); kațru kāu 'especie de fruta o baya con sabor

${ }^{23}$ El diccionario de Starostin et al. (2003) brinda la protoforma que transcribimos con (*).

${ }^{24}$ Este grupo de cognados tiene parecido con el árabe súkkar 'azúcar', el persa shäkär 'azúcar' o el antiguo indio śárkarā' 'grava, grano, azucar' (Klein 1967:1539); es posible que tengan el mismo origen ya que es común, en las lenguas, la polisemia que abarca distintas cualidades de sabor. Tenga en cuenta también que, hasta hace unos pocos siglos, la sal y el ácido eran considerados sustancias de la misma clase - la diferencia recién fue establecida en el siglo XVIII—, incluso la sal era definida como una «sustancia ácida simple» (Roos 2007:3).

${ }^{25}$ Posiblemente se refiera a la Artemisia absinthium que crece en Eurasia y que es usada como especia y también para hacer un licor amargo (Seidemann 2005:52).

${ }^{26}$ Dialecto o lengua de la familia dravídica entre paréntesis. 
picante'; krō 'picante' (kui); xaṛxā 'amargo, picante, altamente salado, áspero'; xarxnā 'afectar al paladar (por cosas saladas o condimentadas en exceso)'; xarxxō 'bilis en su vesícula' (kurukh); qarqe 'amargo'; qátare 'palpitar de dolor' (malto) (Burrow y Emeneau 1984:106).

- Kāram 'cáustico', 'diferentes sales, acritud (como de pimienta)' (malayalam); kāra 'acritud, álcali, cáustico'; karlu 'tierra de sal' (kannada); ka•ra 'picante (como el sabor del curry)' (kodagu); kāra 'sabor u olor picante', 'ardiente'; kāruppu 'una especie de sal fuerte o negra' (tulu); kāru 'salinidad, sal', 'salobre' (telugu) (ibíd.:137).

- Kotuku, kocu, kocuku 'zancudo, mosquito, mosca' (tamil); kotu, kotuku 'zancudo, mosquito' (malayalam); kucune 'zancudo' (irula); kwïf 'especie de insecto mordedor' (toda) (ibíd.:189).

En todos estos grupos de formas similares, se sugiere la asociación entre la sal y cualidades gustativas como lo amargo o ácido; entre sal y el líquido o fermento y entre sal y ciertas especies de vegetales o insectos que pican; obviamente hay razones semánticas para considerar que dichas palabras, en cada instancia, tienen el mismo origen. En la próxima sección, dichos esquemas serán respaldados al tratar de explicar la formación de la palabra quechua para sal.

\section{La palabra quechua: indicios en lenguas arawak}

En quechua, «sal» se expresa con la voz kachi y es el único término que denota el concepto. Es la palabra difundida en todos los dialectos conocidos, desde el quechua central katri /kaĉi/ (BL) hasta el ecuatoriano cachi /kači/ (GR). En vista de que el aimara tiene jayu /hayu/ 'sal', se infiere que no ha tenido influencia de esta otra lengua andina para nombrar a la sal. De esta única raíz quechua se derivan diversos términos, por ejemplo, cachiçapa 'cosa muy salada', cachicani 'echar sal al guisado', cachina ${ }^{27}$ 'tipo de sal para fabricar colores', cachiyan 'hacer sal del agua salada', cachiyoc 'lo que tiene sal' (HOL).

En realidad, la raíz quechua no tiene términos emparentados dentro de los mismos dialectos quechuas; en cambio, en lenguas arawak, existe una gama de términos fonéticamente similares a kachi como la palabra ashéninka catyori 'salado' (PA) o el matsigenka kacho- 'estar muy salado' (SN), además de otros significados asociados a bebedizo e insecto que también observamos en las lenguas asiáticas de la sección 4. Entre estos se encuentran palabras que denotan bebida fermentada, juego venenoso, hormiga o la yuca, que es la materia prima del masato. Elaboramos una lista con dichos cognados y los agrupamos en dos campos semánticos:

${ }^{27}$ Tal vez se refiera al cloruro de estaño. 
Cuadro 1. Cognados de la raíz */kači/ en lenguas arawak

\begin{tabular}{|c|c|c|}
\hline Lengua arawak & $\begin{array}{c}\text { Campo semántico: «sustancia», } \\
\text { "sabor» }\end{array}$ & $\begin{array}{l}\text { Campo semántico: «especie } \\
\text { vegetal o animal», «dolor» }\end{array}$ \\
\hline $\begin{array}{l}\text { asháninka } \\
\text { (KIN) }\end{array}$ & $\begin{array}{l}\text { catsijari 'líquido fermentado o } \\
\text { ácido'; cashitachari 'savia blanca'; } \\
\text { cachoja oni 'estar salado o ácido'; } \\
\text { cachoo- } 27 \text { 'ácido, salado' }\end{array}$ & - \\
\hline ashéninka (PA) & $\begin{array}{l}\text { ocashi 'resina'; cachori 'agrio'; } \\
\text { catyori 'salado' }\end{array}$ & $\begin{array}{l}\text { catsirishi 'especie de planta } \\
\text { picante'; catsicona 'árbol de savia } \\
\text { picante'; caniyatzi 'tallo de yuca'; } \\
\text { catsiquiri 'esp. hormiga'; catsicori } \\
\text { 'esp. hormiga'; catsi- 'doler' }\end{array}$ \\
\hline matsigenka (SN) & $\begin{array}{l}\text {-kashi'resina, líquido venenoso'; } \\
\text { kacho- }^{28} \text { 'estar muy salado, estar } \\
\text { ácido'; kachori 'agrio' }\end{array}$ & $\begin{array}{l}\text { sékatsi 'yuca'; kashirigánire 'esp. } \\
\text { yuca'; katsíkori 'esp. hormiga' }\end{array}$ \\
\hline $\begin{array}{l}\text { nomatsiguenga } \\
\text { (SHA) }\end{array}$ & $\begin{array}{l}\text { cóchóri 'ácido, agrio, fermentado’; } \\
\text { quëchó- 'se fermentó, quëshó- 'se } \\
\text { solidificó' }\end{array}$ & $\begin{array}{l}\text { cochóneri 'esp. hormiga'; catsíberi } \\
\text { 'esp. hormiga'; catsírí 'doloroso, } \\
\text { picante' }\end{array}$ \\
\hline piro yine $(\mathrm{NI})^{29}$ & $\begin{array}{l}\text { kapsagalu 'masato fermentado, sal } \\
\text { amarga'; kapsalu 'amargo, fermen- } \\
\text { tado'; kachgalu 'bebida fermentada'; } \\
\text { kacholu 'ácido, agrio'; kachwugalu } \\
\text { 'picante,' 'licor'; kachwuru 'picante' }\end{array}$ & $\begin{array}{l}\text { kachichi 'curuínse (hormiga)'; kat- } \\
\text { sotalo 'ischimi (hormiga)'; kachinru } \\
\text { 'doloroso'; kachinolu 'dolor' }\end{array}$ \\
\hline resígaro $(\mathrm{AL})$ & kay̆íigí yuca’; kajaákúu 'exprimidor’ & - \\
\hline yukuna (SCH) & - & $\begin{array}{l}\text { kaPachí 'yuca brava (planta y } \\
\text { tronco)'; kachichi?iyú 'hormiga } \\
\text { negra'; kati 'araña' }\end{array}$ \\
\hline piapoco (KLU) & - & àasi 'ají pajarito’ \\
\hline tariana $(\mathrm{AIK})$ & caxiri' 'bebida' & ãsi'pimienta' \\
\hline ignaciano $(\mathrm{OT})$ & $\begin{array}{l}\text { cátsiama 'chicha fuerte, liquido pi- } \\
\text { cante'; -catsi 'sentir ácido, picante'; } \\
\text { ticatsi ácido, agrio' }\end{array}$ & cachiru 'hormiga' \\
\hline lokono $(\mathrm{PE})$ & kasiri 'cerveza de yuca' & - \\
\hline
\end{tabular}

¿Cómo se justifica un supuesto origen común entre la sal y el bebedizo? Se trata indudablemente de una analogía perceptiva: el proceso de transformación (crudococido) de líquido a sustancia concentrada.

Este esquema establece que tanto los brebajes como la sal se someten al mismo proceso de cocción con resultados similares, es decir, produciendo el fermentado,

\footnotetext{
${ }^{28}$ Encuéntrese con el lema cachootaque (cuya segmentación es kačoo-t-ak-e).

${ }^{29}$ Encuéntrese con el lema kachotagantsi (-t- epéntesis y -agantsi 'terminación verbal no finita').

${ }^{30} \mathrm{La}$ abundancia de datos del piro (NI) solo demuestra que hubo aporte de etnias pano.
} 
el concentrado o el coagulado. La sal es, pues, obtenida a través de la cocción de la salmuera en vasijas de arcilla o también se obtiene naturalmente por acción del sol en las salinas de los litorales, en estanques artificiales o en las vertientes de las tierras altas. Aplica también las sales vegetales cuya última etapa comprende la cocción del concentrado lixiviado, técnica recurrente en el interior del Amazonas donde son escasas las salinas naturales. En lenguas del tronco arawak, el iívi' 'sal del monte' del resígaro (AL) que se come con la coca sería, pues, cal obtenida de cenizas vegetales. Del mismo modo, el yukuna jiwi denota una 'sal vegetal hecha de un bejuco' (SCH); el tariana íwi es la sal obtenida de la planta carurú (AIK). Los indígenas del curso superior del Orinoco llaman a la sal chivi, incluyendo a los de Javita quienes hacen el condimento a partir de una especie de palmera o árbol de cacao, no en vano el jugo del fruto del cacao es salino (Humboldt 1907:365).

En consecuencia, el esquema mental de la producción de sal implica un proceso transformativo líquido-sólido o coagulación del concentrado y se asocia, por lo tanto, a la fermentación, a la efervescencia o ebullición, asimismo, a la chicha, el masato o la materia prima de las bebidas ${ }^{31}$. Las palabras son evidencias irrefutables. Observe el siguiente grupo interlingüístico de formas similares en diversas lenguas de la región:

\begin{tabular}{|c|c|}
\hline$p o^{h_{S}}$ & 'sal' (yanesha, DUF) \\
\hline pooch & 'curuhuinse (hormiga)' (yanesha, DUF) \\
\hline$p a^{2} p o^{p 32}$ & 'su espuma, su burbuja' (yanesha, DUF) \\
\hline posu- & 'hediondo, podrido' (piro, NI) \\
\hline posliriri $^{33}$ & 'sal de fruta' (piro, NI) \\
\hline posekalu $^{34}$ & 'fermentable' (piro, NI) \\
\hline$p^{h}$ ojchiy & 'rebosar, fermentar' (quechua, MID) \\
\hline$p^{h}$ Osoko & ‘espuma' (quechua, MID) \\
\hline posoq ${ }^{h} O$ & 'espuma' (quechua, TSC) \\
\hline bosajü & 'fermentar' (orejón, VE) \\
\hline damula & 'sal' (shiwilu, VA) \\
\hline yamora & 'sal' (chayahuita, HA) \\
\hline mamola & 'sal' (chamicuro, PAR) \\
\hline amúra?a- 35 & 'fermentar' (yukuna, SCH) \\
\hline
\end{tabular}

${ }^{31}$ El siguiente extracto de un mito siona retrata esta asociación sal-masato: «Después Baina dijo a sus esposas que hagan chicha de maíz y que pongan un poco de masato (el concentrado que se mezcla con agua para hacer chicha) en cada trocha (este fue el origen de los lamederos de sal). En un sendero solo han comido venados, pero otro ha sido visitado por la danta» (Vickers 1989:161).

${ }^{32}$ Conformado por el prefijo posesivo de tercera persona pa?- y la raíz - po? 'espuma'.

${ }^{33}$ Conformado por la raíz pos, el nominalizador participial -liri y el sufijo de genero -ri (Hanson 2010; Nies 1986).

${ }^{34}$ Conformado por la raíz * pose, el asertivo - $k a$ y el nominalizador o atributivo -lu (Hanson 2010; Nies 1986).

${ }^{35}$ Encuéntrese como amúra'atakaje, el cual está conformado por la terminación verbal 
Posiblemente, la palabra quechua yacu 'agua, agua hirviente' (HOL) esté relacionado con el nombre de la sal en chipaya, yaku (CER). Aunque no se descarta que sea solo un producto metonímico a partir de la expresión «río/agua de sal», como parece ser el caso de la palabra candoshi yomi 'sal' (TU), a partir del aguaruna yúmi 'agua' (DEI).

Ahora bien, respecto a la asociación sal-insecto ¿cuál sería el origen semántico de la relación entre la sal y los insectos que pican? Esta sería una asociación indirecta intercedida por la imagen del bebedizo, un alimento potencialmente venenoso que quema la boca y el estómago cuando tiene propiedades astringentes o sabor picante; por ejemplo, el masato se percibe como picante cuando se exacerba después del cuarto día (Daggett 1983:303). El adjetivo kukama-kukamiria tai se define así: «ser picante, puede ser picante natural como el ají o se extiende para el masato cuando pasa más de 4 días» (Vallejos y Amías 2015). Por lo tanto, el nombre de las bebidas fermentadas — vinculado al nombre de la sal — se habría extendido al concepto de los insectos que pican. Sin embargo, podría tratarse de una asociación directa salinsecto si tomamos en cuenta las propiedades cáusticas de la sal vegetal con alto contenido de iones potasio y bajo contenido de sodio, cuyo sabor es acre y picante ${ }^{36}$ (Echeverri 2001:398). Según Echeverri, los uitotos vinculan la sal vegetal con los animales venenosos tales como insectos que pican, serpientes, pero también plantas espinosas o urticantes (ibíd.:405). Si tomamos en cuenta la escasez de depósitos de sal en el interior de la Amazonía, entonces, muchos nombres de sal amazónicos refieren no al cloruro de sodio, sino a estos residuos sólidos y picantes extraídos de las cenizas vegetales tal como vimos en la sección 3 .

Con frecuencia encontramos este patrón, sal-insecto, en los vocabularios de lenguas amazónicas, como por ejemplo:

(2) móã 'sal' (tuyuca, BAR)

ẽmoã 'esp. de oruga venenosa ${ }^{37}$, (hormiga) magiñás ${ }^{38}$ ' (tuyuca, BAR)

wee 'sal' (huambisa, JAK)

wee ete 'esp. de avispa' (huambisa, JAK)

week '(hormiga) curuhuinse' (huambisa, JAK)

töô 'sal' (cashinahua, AB)

to?ok 'taoca (hormiga)' (kaapor, KAK)

toi 'hormiga cazadora' (sirionó, GAS)

-kaje (nominalizador) y el segmento -ta que sería el sufijo causativo, por lo que la raíz primigenia habría sido amúra?a-.

${ }^{36}$ En más de una ocasión los cronistas afirman que la sal [vegetal] de los nativos amazónicos tiene sabor a salitre (Roth 1924:223; Heriarte 1874:49).

${ }^{37}$ Según la definición, esta oruga tiene pelos urticantes cuyo escozor permanece por muchas horas (Barnes 2012:141).

${ }^{38}$ En los diccionarios, siempre se resalta la agresividad de esta hormiga majiñá o magiñá (nombre regional), es decir, es una hormiga propensa a picar. 
Viniendo al caso, nos preguntamos si la raíz tupí-guaraní */tasi/ */taši / 39 que denota cierta especie de hormiga estaria relacionada con el nombre de sal en lenguas pano, tashi /taši/. De ser así, implicaría que el nombre de la sal en pano designaba originalmente a una sal de origen vegetal. Más bien, el término pano tashi, así como los datos del corpus (2), tendrían otro desarrollo léxico-semántico que serán explicados en la siguiente sección.

\section{Planteamiento de la derivación de la palabra quechua kachi}

Nuestra hipótesis se apoya en la asociación sal-bebedizo en donde se hace notorio el proceso transformativo de la materia prima en bebida concentrada. Este esquema, además, permite abarcar todo tipo de sal que se somete a cocción en la última etapa de producción, incluyendo a la sal gema que los nativos hierven para ablandar. Para reforzar esta hipótesis, aducimos pruebas del mismo sistema léxicosemántico en lenguas arawak, tupí-guaraní y pano. Veamos.

La palabra más común para sal en lenguas $\operatorname{arawak}^{40}$ es tzivi (PA) o tibi (SHA), cuya protoforma sería */tsi $\beta \mathrm{i} /$ ' 'sal'. Esta estaría compuesta de dos partes, siendo el primer segmento una primitiva raíz presente en diferentes lenguas de la familia: */tsi/. Esta corta raíz habría significado líquido coagulado; por ejemplo, en el ashéninka, -she $\sim$-shi '(líquido) espeso' (PA); en el yanesha, che'n- 'coagular' (DUF); en nomatsiguenga, -se '(líquido) en forma de pasta' (SHA). También está presente en lenguas de otras familias linguísticas, como el tupí-guaraní nheêngatú ty /tì/ 'zumo, licor, salsa' (STR) o el guaraní tĭ 'agua, zumo, caldo' (RM). En el cashinahua, encontramos la raíz ti 'resina' (AB), tsi 'líquido coagulado' (MON), de la que se deriva tiwa 'exprimir' (AB), tsi va'exprimir' (MON), palabra que recuerda a la forma arawak. Ciertamente, la palabra arawak tzivi 'sal' sería cognado con la bebida nativa chibét ${ }^{4}$, extendida en gran parte de Brasil, hecha de harina de mandioca (Monteiro 1963:58). De hecho, la extracción del jugo venenoso de la mandioca implica un proceso de filtración del líquido tal como ocurre en la lixiviación de la ceniza vegetal. Un indicio de que la sal vegetal era la sal típica de los antiguos arawak.

La palabra para sal en lenguas tupí-guaraníes es ejemplificado, entre otros, por yuquй en el guaraní misionero (RM); iukyra en el nheêngatú (STR); jukyr en el ka'apor (KAK) o embikesa ${ }^{42}$ en el siriono (GAS), cuya raíz despejada es -ke-. Esta última parece no ajustarse con la protoforma reconstruida *jukit 'sal'

\footnotetext{
${ }^{39}$ Algunos ejemplos: taç̌̆ 'enfermedad, dolor, hormiga' (RM); tasi 'hormiga', 'doler' (PRI); taxy 'esp. hormiga', acy 'veneno' (STR); tsatsiwa 'hormiga' (VAL).

${ }^{40}$ Algunos nombres de sal son $t u w u$ /ti $\beta \mathrm{i} /$ en piro (NI); iwi /ißi/ en tariana (AIK); -cheve/ čeße/en ignaciano (OT).

${ }^{41}$ El chibé es una bebida nativa, extendido en gran parte de Brasil, hecho de harina de mandioca (Monteiro 1963:58).

${ }^{42}$ La palabra embikesa está formada por el prefijo causativo $m b i$-, la raíz -ke- y el nominalizador $-s a$.
} 
(Meira 2015) si consideramos que - ke- era en realidad un verbo como constatado en el guaraní misionero mediante la palabra que /ke/ 'dormir, reposar' (RM), por extensión, connotando a la sustancia cuajada después de haber reposado: oque

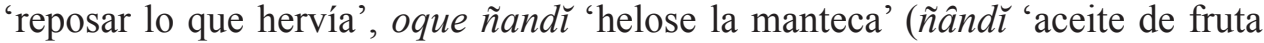
o árbol, grasa'), ei oque 'está la miel asentada después de hervir' (RM). Por lo tanto, -ke- no sería una raíz heredada del tronco tupí-guaraní, el cual se evidencia en otras lenguas foráneas como el yanesha, lengua arawak, mediante el término $p a^{2} q u e^{243} / p a^{3}-k e^{2} /$ 'su cuajo o cuajada de yuca' (DUF); en el piapoco, mediante el término nuquini ${ }^{44} / n u-k i-n i /$ 'mi yuca' o quinía 'jugo no cocido de yuca brava' (KLU). El dato del proceso de reposo es revelador porque nos informa como el último paso de la preparación es el más importante para los nativos ${ }^{45}$. Hay más datos que indicarían una correlación entre el verbo reposar y el coagulado en lenguas arawak: en yanesha mas- 'coagularse' (DUF) que es la misma raíz del verbo ashéninka mag- 'dormir' (PA), del tariana -ma 'dormir' (AIK) o del ignaciano -ímaca 'dormir' (OT). El verbo, en el yanesha, es mu- 'dormir' (DUF) similar al resígaro -ímú 'dormir' (AL), del cual se derivó el verbo estativo ka?múú? 'estar fermentado' (AL). En tariana, el verbo whé whéta 'asentar' está relacionado con el verbo -whéta 'fermentar' (AIK), por lo tanto, esta sería en realidad el significado original, tanto del nombre de sal del huambisa, wee, como del nombre de la hormiga week (JAK), véase el corpus (2). El siguiente escenario ilustra la operación expresada con el verbo tariana -wheta: dira-karu-pena-nuku du-nua du-pala du-yana du-wheta 'cocinaba la bebida de él (el chibé), colocaba (en la olla), dejaba asentar allí' (Aikhenvald 2001:235).

El nombre de sal en lenguas pano, tashi, se habría formado por composición de la raíz arawak */tsi/, tal como el cashinahua tsi 'líquido coagulado"46 $(\mathrm{MON})$, y una antigua raíz $* t a$, la cual se manifiesta sugestivamente en la palabra cashinahua taka 'cuajado' (AB). Posiblemente, el término cashinahua tae 'sal' de R\&T sea apocopé de taka. Lo interesante es que la lengua aimara parece conservar la raíz pano * $t a$ en diversas palabras derivadas cuyos significados corresponden a los ya

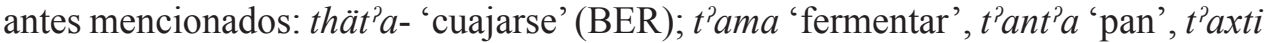
'savia, jugo', tikt'a 'coagularse' (HUA).

Ahora bien, en el caso del quechua, encontramos que los términos o raíces quechuas que denotan esta terminología relacionada al bebedizo o el líquido coagulado no tienen similitud fonética con la palabra kachi 'sal'. Por ejemplo, ttucurun 'cuajarse algo de comer', ticayarin 'cuajarse la leche o sebo', ppoccocun 'leudarse' (HOL); t'ucuru 'cosa cuajada' (TSC); ticayay 'volverse sólida una cosa líquida', pokoy 'madurar, fermentar', $p^{h}$ ojchiy 'rebosar, fermentar' (MID); togush

\footnotetext{
${ }^{43}$ Formado por pa?- 'prefijo posesivo 3P' y $-k e$ ? 'cuajado de yuca'.

${ }^{44}$ Formado por el prefijo posesivo $n u$-, la raíz - $k i$ - 'yuca' y el marcador posesivo alienable $-n i$.

${ }^{45}$ Echeverri (2001) dedica un capítulo de su ensayo de la sal vegetal a la fase del cuajado.

${ }^{46}$ Como idea sugestiva, */tsi/ estaría también presente en palabras como el cashinahua tsi 'líquido coagulado' (MON).
} 
'papas fermentadas', pogun 'madurar, [fermentar]' (WE). Estas raíces quechuas,

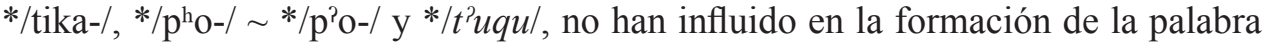
kachi; no obstante, se manifiestan en otras lenguas de la región como es el caso del aimara tikt'a 'coagularse' (HUA), el orejón bosajü 'fermentar' (VE), el cashinahua töi 'cuajar, espesar la bebida' y töô 'sal' (AB).

La palabra quechua $k a c h i$, por lo tanto, no sería originalmente quechua, sino una formación de origen arawak. Kachi estaría conformada, en su segunda sílaba, por la raíz */tsí/, deducida a partir de */tsi $\beta_{\tau} \mathbf{i} /$ 'sal'. La otra parte, es decir, el segmento con sonido oclusivo velar, también parece estar presente en el mismo grupo arawak, por ejemplo, kaádé 'espuma', kaání 'yuca brava', kamóó- 'bebida fermentada', ka?ma'fermentar', ka?múú? 'estar fermentado' (AL); káini 'manicuera'47, kána 'maíz' (AIK). En efecto, todas estas palabras contienen una primitiva raíz */ka?/ cuyo significado habría sido 'fermentar, cuajar, reposar'. Esta raíz ligada a */tsi/ habrían formado no solo al quechua kachi, sino también a las demás palabras del Cuadro 1: quëchó- /kcečo/ 'se fermentó' (SHA); kaǰrigi 'yuca' (AL); caxiri 'bebida' (AIK); cátsiama 'chicha fuerte' (OT). Evidentemente, el quechua aka 'chicha' (TSC) o $a q^{h} a$ 'bebida fermentada' (MID) es una reminiscencia de la antiquísima raíz */ka?/. Así también, la raíz arawak estaría presente en lenguas jivaroanas, por ejemplo, kantekú 'espeso, cuajado', kajüt 'fermentar', kánut 'dormir' (LAR); kariatsa, kariamu 'fermentar', kánamu, kánut 'dormir' (JAK); kãẽngtin 'coagularse', karíatin 'fermentar, agriarse', kajíngtin 'dormir' (FAS). Tessmann ([1930] 1999) reportó la palabra katsi $\sim$ atsi 'sal de piedra' en zona de los jívaros, nombre que era usado para distinguir la sal gema de la sal vegetal.

\section{Conclusiones}

Las consecuencias de la presente propuesta no solo son de índole lingüística, sino también sugiere algunas ideas sugerentes respecto a la etnohistoria.

En primer lugar, la palabra para sal del quechua no fue un simple préstamo arawak, sino una formación estructural, compuesta por raíces separadas que han influenciado en otras lenguas del territorio andino-amazónico. La formación de la palabra quechua parece implicar un prolongado tiempo de contacto desde el cual también alcanzó a los jívaros del norte del Perú, quienes tenían comunicación con la selva central a través del Huallaga. Así, el hipotético y original significado de $k a c h i<* / \mathrm{ka}$ / + */tsi/ habría sido «líquido reposado» o «cuajado» que abarca no solo a la preparación de sal, sea mineral o vegetal, sino también a cualquier tipo de bebedizo que se somete a fermentación y cocción. Una unidad léxica por sí misma no necesita mucho tiempo para que sea adoptada por una comunidad extranjera, mientras que un fragmento gramatical o morfema requiere mucho más tiempo para que sea adoptado por diferentes lenguas receptoras y en más de un tipo de categoría gramatical. Así también, existiría otra conexión amazónica de índole lingüística en el término quechua wasi 'casa'48 que sería una reanálisis del verbo arawak / $\beta$ aši-/

\footnotetext{
${ }^{47}$ Especie de mandioca o bebida de mandioca.

${ }^{48}$ Artículo en preparación, acerca de la etimología de la palabra quechua wasi.
} 
'cubrir con hojas en forma de techo' (SN) o del nombre */waš-/ 'techo de palmera' (NI). La cantidad de datos lingüísticos, apuntan a una herencia más amazónica que propiamente andina de los antiguos quechuas.

Esta propuesta lingüística tiene, por lo tanto, implicaciones históricas relacionadas a la expansión de grupos influyentes que vinieron desde el interior del continente. En efecto, la llegada de los prehistóricos grupos arawak ascendiendo por el Purús recuerda a la expansión de los bandeirantes tratando de alcanzar la cordillera andina (Rosas 2008:107). Uno de estos grupos abrió la ruta hacia occidente al descubrir el Urubamba y luego ascendiendo por el Tambo y Perené, hasta alcanzar tierras ricas en sal de roca y ríos salados. Como se deduce de la sección 2, el Ucayali, el Huallaga y la selva central formaron puntos estratégicos del comercio de sal amazónico donde grupos foráneos bajaban desde las cabeceras fluviales hasta Loreto, para extraer o comerciar sal; mientras que por el centro, estrechamente conectado con la selva norte peruana a través de los ríos Huallaga y Ucayali, los arawak comerciaban sal con grupos que venían desde el Purús y Madre de Dios. Pronto la zona se convirtió en una esfera de acción de intercambio de bienes y conocimientos, influencia cultural que se habría extendido hacia todos los puntos cardinales dentro del territorio peruano. Esto no implica que los grupos originarios desconocían la técnica de la fermentación y la coagulación, más bien los protoarawak habrían contribuido a mejorar el aspecto tecnológico y económico de los bienes explotados en los grupos étnicos originarios, por lo tanto, no se descarta que la presencia arawak en la selva central peruana haya fomentado un mayor comercio de sal en la región, lo que explicaría por qué las palabras para sal en lenguas quechua, jivaroana y pano estén formadas por antiguas raíces arawak.

En realidad, una importante interacción entre andinos y amazónicos subandinos se estuvo llevando a cabo durante siglos desde hace más de 2000 años como señala Hornborg y Eriksen (2011:139) y tomando en cuenta, además, que los preprotoquechua habrían llegado a la sierra central peruana a finales del Horizonte Temprano (200 a. C.) y conquistaron a los pre-protoaimaras originarios del lugar (Adelaar 2010). Surge la pregunta ¿de dónde vinieron estos ancestros quechuas? Todo indica que ellos procederían del este, tal vez azuzados por los protoarawak subandinos para invadir tierras hacia el oeste, en el interior de los Andes, debido a que los pano mayoruna tomaron ventaja hacia el norte, en el curso inferior del Ucayali, como indica Fleck (2013:21).

En suma, desde hace más de dos milenios estuvieron conviviendo diversas culturas en un mismo territorio, atrayéndose y rechazándose entre sí, e impulsando relaciones areales. Los múltiples términos de sal yanesha ${ }^{49}$ parece ser un vestigio de la antigua confluencia étnica, en donde la zona del Perú central se convirtió en el espacio hegemónico de la explotación de la sal. Por otro lado, hay notables indicios de un protagonismo pano a través de la información etnográfica y lingüística que establece su foco inicial en el alto Ucayali y que coincide con el control de los depósitos de sal de los cerros y riachuelos en dicha cuenca. La presunta conexión

${ }^{49}$ Algunos términos de sal yanesha son pos, carob, pesharrem, po?yarr (DUF). 
prehistórica pano-aimara que propusimos en la sección 6 es solo lingüística, por lo que solo se mantiene a la expectativa hasta ampliar la investigación del tronco aimaraico.

\section{Referencias}

Abreu, João Capistrano de. 1914. Rã-txa hu-ni-ku-̃̃: a lingua dos caxinauás do Rio Ibuaçu affluente do Muru. Rio de Janeiro: Typographia Leuzinger.

Adelaar, Willem. 2010. "Trayectoria histórica de la familia lingüística quechua y sus relaciones con la familia lingüística aimara." Boletín de Arqueología PUCP 14: 239-254.

Aikhenvald, Alexandra Y. 2001. "Dicionário tariana - português e português tariana." Boletim do Museu Paraense Emílio Goeldi 17 (1): 3-388.

.2015. “Amazonia: Linguistic History." En The Global Prehistory of Human Migration, editado por Peter Bellwood e Immanuel Ness, 384-391. Chichester: Wiley Blackwell.

Aikhenvald, Alexandra Y. y Robert M. W. Dixon. 1999. “ $\quad$ Other Small Families and Isolates." En The Amazonian Languages, editado por Robert M. W. Dixon y Alexandra Y. Aikhenvald, 341-384. New York: Cambridge University Press.

Allin, Trevor. 1979. Vocabulario resígaro. Lima: Instituto Lingüístico de Verano. https://www.sil.org/system/files/reapdata/40/14/30/40143023256164218758156 341497533559504/dt16.pdf

Arriaga, Pablo Joseph de. 1621. Extirpacion de la idolatria del Piru. Lima: Geronymo de Contreras.

Barnes, Janet. 2012. Diccionario bilingüe tuyuca-español, español-tuyuca. Bogotá: Fundación para el Desarrollo de los Pueblos Marginados.

Beekes, Robert. 2010. Etymological Dictionary of Greek. Vol. 1. Leiden: Brill.

Bertonio, Ludovico. [1612] 1879. Vocabulario de la lengua aymara. Leipzig: B. G. Teubner.

Black, Nancy. 1990. Lecciones para el aprendizaje del quechua del sureste de Pasco y el norte de Junín. Lima: Dirección Departamental de Educación-Pasco/ Instituto Lingüístico de Verano.

Burrow, Thomas y Murray B. Emeneau. 1984. A Dravidian Etymological Dictionary. Oxford: Clarendon Press.

Buse, Hermann. 1975. Historia marítima del Perú: Época prehistórica. Tomo II, vol. 1 y 2. Lima: Instituto de Estudios Histórico-Marítimos del Perú.

Castellanos, Juan de. 1886. Historia del Nuevo Reino de Granada. Tomo I. Madrid: Imprenta de A. Pérez Dubrull.

Cerrón-Palomino, Rodolfo. 2010. "Contactos y desplazamientos lingüísticos en los Andes centro-sureños: el puquina, el aimara y el quechua." Boletín de Arqueología PUCP 14: 255-282.

Cerrón-Palomino, Rodolfo y Enrique Ballón. 2011. Chipaya: Léxico - 


\section{Etnotaxonomía. Lima: Fondo Editorial PUCP.}

Cipolletti, María Susana y Fernando Payaguaje. 2008. La fascinación del mal. Historia de vida de un shamán secoya de la Amazonía ecuatoriana. Quito: AbyaYala.

Cobo, Bernabé. 1890. Historia del Nuevo Mundo. Tomo I. Sevilla: E. Rasco.

Daggett, Carole. 1983. "Las funciones del masato en la cultura chayahuita." Antropológica PUCP 1: 301-310.

Dahl, Östen, Christopher Gillam, David G. Anderson, José Iriarte y Silvia M. Copé. 2011. "Linguistic Diversity Zones and Cartographic Modeling: GIS as a Method for Understanding the Prehistory of Lowland South America." En Ethnicity in Ancient Amazonia, editado por Alf Hornborg y Jonathan D. Hill, 211-224. Boulder: University Press of Colorado.

D'altroy, Terence N. 2015. The Incas. Malden: Blackwell.

Deicat, Gerardo Wipio. 1996. Diccionario aguaruna-castellano castellanoaguaruna. Lima: Instituto Lingüístico de Verano.

Diniz, Marco Túlio Mendonça y Fábio Perdigão Vasconcelos. 2016. "Porque o nordeste produz sal marinho? Estudo analógico do potencial do clima." Caderno de Geografia 26 (2): 355-379.

Diniz, Marco Túlio Mendonça, Fábio Perdigão Vasconcelos y Márcia Barbosa Martins. 2015. "Inovação tecnológica na produção brasileira de sal marinho e as alterações sócioterritoriais dela decorrentes: uma análise sob a ótica da teoria do empreendedorismo de Schumpeter." Sociedade \& Natureza, Uberlândia 27 (3): 421-438.

Duff-Tripp, Martha. 1998. Diccionario yanesha' (amuesha)-castellano. Lima: Instituto Lingüístico de Verano. https://www.sil.org/system/files/ reapdata/14/32/53/143253772569919515318148084495238031876/slp47.pdf

Echeverri, Juan Alvaro. 2000. "The First Love of a Young Man: Salt and Sexual Education among the Uitoto Indians of Lowland Colombia." En The Anthropology of Love and Anger, editado por Joanna Overing y Alan Passes, 33-45. London: Routledge.

Echeverri, Juan Alvaro. 2001. 'La sal de monte: un ensayo de 'halofitogenografía' uitoto." En Imani Mundo: Estudios en la Amazonía colombiana, editado por Carlos Franky y Carlos Zárate, 397-477. Bogotá: Unibiblos.

Echeverri, Juan Alvaro y Oscar Román-Jitdutjaaño. 2011. "Witoto ash salts from the Amazon." Journal of Ethnopharmacology 138: 492-502.

Fast Mowitz, Gerhard, Ruby Warkentin y Daniel Fast. 1996. Diccionario achuar-shiwiar - castellano. Yarinacocha: Instituto Lingüístico de Verano. https://www.sil.org/system/files/ reapdata/15/64/89/156489401245172331263552509065358250150/slp36.pdf 
Fleck, David W. 2013. Panoan Languages and Linguistics. New York: American Museum of Natural History.

Gasparini, Noé y Victor Hugo Dicarere Mendez. 2015. Diccionario siriono. Trinidad: Tiempos del Beni.

Grimm, Juan. 1897. La lengua quichua (dialecto de la República del Ecuador). Friburgo de Brisgovia: B. Herder.

González Holguín, Diego. 1608. Vocabulario de la lengua general del Perú llamada qquichua o lengua del Inca. Lima: Francisco del Canto.

Hanson, Rebecca. 2010. "A Grammar of Yine (Piro)." PhD dissertation, La Trobe University, Bundoora.

Hart, Helen. 1988. Diccionario chayahuita-castellano. Lima: Instituto Lingüístico de Verano. https://www.sil.org/system/files/reapdata/53/83/82/ 53838 289028662789570876716126619509316/slp29.pdf

Hemming, John. 1978. Red Gold. The Conquest of the Brazilian Indians. Cambridge: Harvard University Press.

Heriarte, Mauricio de. 1874. Descripção do estado do Maranhão, Pará, Corupá e rio das Amazonas. Vienna: Imprensa do filho de Carlos Gerold.

Herrera, Antonio de. 1728. Historia general de las Indias Ocidentales. Tomo II. Amberes: Juan Bautista Verdussen.

Hornborg, Alf y Love Eriksen. 2011. “An Attempt to Understand Panoan Ethnogenesis in Relation to Long-Term Patterns and Transformations of Regional Interaction in Western Amazonia." En Ethnicity in Ancient Amazonia, editado por Alf Hornborg y Jonathan D. Hill, 129-151. Boulder: University Press of Colorado. Huayhua Pari, Felipe. 2009. Diccionario bilingüe polilectal: aimara-castellano, castellano-aimara. Lima: Fondo Editorial UNMSM.

Humboldt, Alexander von y Aimé Bonpland. 1907. Personal Narrative of Travels to the Equinoctial Regions of America. Vol. II. London: George Bell \& Sons.

Izaguirre, Bernardino. 1926. Historia de las misiones franciscanas y narración de los progresos de la geografía en el oriente del Perú. Vol. 12. Lima: Talleres Tipográficos de la Penitenciaría.

Jakway, Martha. 1987. Vocabulario huambisa. Lima: Instituto Lingüístico de Verano. https://www.sil.org/system/files/reapdata/15/45/69/154569950922777224 740467572055985243548/slp24.pdf

Jiménez de la Espada, Marcos. 1879. Tres relaciones de antigüedades peruanas. Madrid: Imprenta y Fundición de M. Tello.

Jiménez de la Espada, Marcos. 1881. Relaciones geográficas de Indias, Perú. Tomo I. Madrid: Tipografía de Manuel G. Hernández.

Jiménez de la Espada, Marcos. 1885. Relaciones geográficas de Indias, Perú. Tomo II. Madrid: Tipografía de Manuel G. Hernández.

Jiménez de la Espada, Marcos. 1897. Relaciones geográficas de Indias, Perú. Tomo III. Madrid: Tipografía de los hijos de M. G. Hernández.

Kakumasu, James Y. y Kiyoko Kakumasu. 2007. Dicionário por tópicos kaapor - 
português. Cuiabá: Associação Internacional de Lingüística.

Karsten, Rafael. [1935] 2000. La vida y la cultura de los shuar. Quito: Abya-Yala.

Kindberg, Lee. 1980. Diccionario asháninca. Yarinacocha: Instituto Lingüístico de Verano. https://www.sil.org/system/files/reapdata/ 15/05/87/150587103864951 424705632321744367293106/dt19.pdf

Klein, Ernest. 1966/1967. A Comprehensive Etymological Dictionary of the English Language. 2 vols. Amsterdam: Elsevier Publishing Company. "”

Koch-Grünberg, Theodor. 1913. "Betóya-Sprachen Nordwestbrasiliens und der angrenzenden Gebiete." Anthropos 8 (6): 944-977.

Larson, Mildred L. 1966. Vocabulario aguaruna de Amazonas. Lima: Instituto Lingüístico de Verano. https://www.sil.org/system/files/reapdata/ 59/72/83/597 28398026071460917794592167972564882/slp03.pdf

Lino Samanamud, Teodoro. 1962. "Las salinas del Perú." Tesis para obtener el grado de ingeniero de minas, Universidad Nacional de Ingeniería, Lima.

Majno, Guido. 1991. The Healing Hand: Man and Wound in the Ancient World. Cambridge: Harvard University Press.

Middendorf, Ernst W. 1890. Wörterbuch des Runa Simi oder der Keshua-Sprache. Leipzig: F. A. Brockhaus.

Montag, Susan. 1981. Diccionario cashinahua. Yarinacocha: Instituto Lingüístico de Verano. https://www.sil.org/system/files/reapdata/ 11/98/57/1198575542358 13427595875320394596487807/slp09.pdf

Monteiro, Mário Ypiranga. 1963. "Alimentos preparados à base da mandioca." Revista Brasileira de Folclore 3 (5): 37-82.

Moseley, Michael E. 2001. The Incas and their Ancestors: The Archaeology of Peru. London: Thames \& Hudson.

Nies, Joyce. 1986. Diccionario piro (yine). Lima: Instituto Lingüístico de Verano. https://www.sil.org/system/files/reapdata/ 15/70/15/1570158522372741909454 83703959623321712/slp22.pdf

Ott, Willis y Rebecca Burke de Ott. 1983. Diccionario ignaciano y castellano. Cochabamba: Instituto Lingüístico de Verano. https://www.sil.org/system/files/ reapdata/75/70/42/75704259799025203588472766406243636083/18865.pdf

Parker, Stephen. 1987. Vocabulario y textos chamicuro. Lima: Instituto Lingüístico de Verano. https://www.sil.org/system/files/reapdata/ 11/78/52/1178521807560 30133865083735493503102914/ccp21.pdf

Patiño, Víctor Manuel. 1992. Historia de la cultura material en la América equinoccial. Tomo V. Bogotá: Instituto Caro y Cuervo.

Payne, David. 1980. Diccionario ashéninca-castellano. Lima: Instituto Lingüístico de Verano. https://www.sil.org/system/files/reapdata/ 27/59/65/2759650278240 6212904632085133341587136/dt18.pdf 
Pet, Willem Jan Agricola. 1987. "Lokono Dian, the Arawak Language of Suriname: A Sketch of its Grammatical Structure and Lexicon." PhD dissertation, Cornell University, Ithaca.

Pharris de Klumpp, Deloris A. 1995. Vocabulario piapoco-español. Bogotá: Instituto Lingüístico de Verano. https:/www.sil.org/system/files/reapdata/ 13/ 12/66/131266150671347557890177502100769298217/VocPiapoco_36065.pdf

Poeppig, Eduard. 1836. Reise in Chile, Peru, und auf dem Amazonenstrome während der Jahre 1827-1832. Tomo II. Leipzig: Friedrich Fleischer/J. C. Hinrichssche Buchhandlung.

Raimondi, Antonio. 1874. El Perú. Tomo I. Lima: Imprenta del Estado.

Raimondi, Antonio. 1876. El Perú. Tomo II. Lima: Imprenta del Estado.

Renard-Casevitz, France-Marie. 1994. "La sal: mujer gema, mujer condimento." Revista de Antropología, UNMSM 1 (1): 81-101.

Roos, Anna Marie. 2007. The Salt of the Earth. Natural Philosophy, Medicine, and Chymistry in England, 1650-1750. Leiden: Brill.

Rosas Moscoso, Fernando. 2008. Del Río de la Plata al Amazonas: El Perú y el Brasil en la época de la dominación ibérica. Lima: Universidad Ricardo Palma, Editorial Universitaria.

Roth, Walter Edmund. 1924. An Introductory Study of the Arts, Crafts, and Customs of the Guiana Indians. Thirty-Eight Annual Report of the Bureau of American Ethnology 1916-1917. Washington: Government Printing Office.

Ruiz de Montoya, Antonio. 1876. Vocabulario y tesoro de la lengua guarani o mas bien tupi. Viena: Faesy y Frick.

Schauer, Stanley, Junia Schauer, Eladio Yucuna y Walter Yucuna. 2005. Diccionario bilingüe: yukuna-español, español-yukuna. Bogotá: Editorial Fundación para el Desarrollo de los Pueblos Marginados.

Seidemann, Johannes. 2005. World Spice Plants. Economic Usage, Botany, Taxonomy. Berlin: Springer-Verlag.

Shaver, Harold. 1996. Diccionario nomatsiguenga-castellano, castellanonomatsiguenga. Yarinacocha: Instituto Lingüístico de Verano. https://www.sil. org/system/files/reapdata/ 15/88/18/1588180072114704372726071230489945 21639/slp41.pdf

Snell, Betty A. 2011. Diccionario matsigenka - castellano. Lima: Instituto Lingüístico de Verano. https:/www.sil.org/system/files/reapdata/ 18/56/85/185 68503648705946088000241499027872639/mcbDIC_web.pdf

Sousa, Gabriel Soares de. 1879. Tratado descriptivo do Brasil em 1587. Rio de Janeiro: Typographia de João Ignacio da Silva.

Starostin, Sergei, Anna Dybo y Oleg Mudrak. 2003. Etymological Dictionary of the Altaic languages. Leiden: Brill. 
Stradelli, Ermano. 1929. "Vocabularios da lingua geral portuguez-nheêngatú e nheêngatú-portuguez, precedidos de um esboço de Grammatica nheêngaumbuê-sáua mirî e seguidos de contos em lingua geral nheêngatú poranduua." Revista do Instituto Historico e Geographico Brasileiro 104 (158): 9-768.

Tessmann, Günter. [1930] 1999. Los indígenas del Perú nororiental. Quito: AbyaYala.

Tuggy, John. 1966. Vocabulario candoshi de Loreto. Yarinacocha: Instituto Lingüístico de Verano. https:/www.sil.org/system/files/reapdata/ 52/33/34/523 33475898881350721713363142485149242/slp02.pdf

Vaan, Michiel de. 2008. Etymological Dictionary of Latin and the Other Italic Languages. Leiden: Brill.

Valenzuela, Pilar. 2013. Diccionario shiwilu-castellano, castellano-shiwilu. Lima: Federación de Comunidades Nativas de Jeberos.

Vallejos Yopán, Rosa y Rosa Amías Murayari. 2015. Diccionario kukamakukamiria castellano. Iquitos: Programa de Formación de Maestros Bilingües de la Amazonía Peruana/Asociación Interétnica de Desarrollo de la Selva Peruana/ Instituto Superior de Educación Público Loreto.

Vázquez de Espinosa, Antonio. 1948. Compendio y descripción de las Indias Occidentales. Washington: Smithsonian Institution.

Velie, Daniel y Virginia Velie. 1981. Vocabulario orejón. Lima: Instituto Lingüístico de Verano. https://www.sil.org/system/files/reapdata/ 21/44/30/2144304951012 9354652194008231235696793/slp16.pdf

Von Tschudi, Johann Jakob. 1853. Die Kechua-Sprache: Wörterbuch. Wien: Kaiserlich-Königlichen Hof- und Staatsdruckerei.

Wallace, Alfred Russel. 1889. Travels on the Amazon and Rio Negro. London: Ward, Lock \& Co.

Weber,DavidJohn,Félix Cayco, TeodoroCaycoy MarleneBallena. 1998. Rimaycuna: Quechua de Huánuco. Lima: Instituto Lingüístico de Verano. https://www.sil.org/ system/files/reapdata/17/21/05/1721055302901112437222763476002933213/ slp48.pdf

Weiss, Gerald. 1975. "Campa Cosmology. The World of a Forest Tribe in South America." Anthropological Papers of the American Museum of Natural History 52 (5): 217-588.

Wörrle, Bernhard. 1999. De la cocina a la brujería: La sal entre indígenas y mestizos en América Latina. Quito: Abya-Yala.

\section{Abreviaturas de diccionarios}
$\mathrm{AB}$
cashinahua (pano), Abreu, 1914
AIK
tariana (arawak), Aikhenvald, 2001 
AL resígaro (arawak), Allin, 1979

BAR tuyuca (tucano), Barnes, 2012

BER aimara, Bertonio, 1879

BL quechua de Pasco y Junín, Black, 1990

CER chipaya (uru-chipaya), Cerrón-Palomino \& Ballón, 2011

DEI aguaruna (jivaroana), Deicat, 1996

DUF yanesha, Duff-Tripp, 1998

FAS achuar-shiwiar (jivaroana), Fast Mowitz et al., 1996

GAS sirionó (tupí-guaraní), Gasparini \& Dicarere, 2015

GR quechua, Grimm, 1897

HA chayahuita (cahuapana), Hart, 1988

HOL quechua, González Holguín, 1608

HUA aimara, Huayhua, 2009

JAK huambisa (jivaroana), Jakway, 1987

KAK kaapor (tupí-guaraní), Kakumasu \& Kakumasu, 2007

KIN asháninka, Kindberg, 1980

KLU piapoco (arawak), Pharris de Klumpp, 1995

LAR aguaruna (jivaroana), Larson, 1966

MID quechua Middendorf, 1890

MON cashinahua, Montag, 1981

NI piro (yine), Nies, 1986

OT ignaciano (arawak), Ott \& Ott, 1983

PA ashéninka, Payne, 1980

PAR chamicuro (arawak), Parker, 1987

PE lokono (arawak), Pet, 1987

PRI sirionó (tupí-guaraní), Priest \& Priest, 1985

RM guaraní misionero (tupí-guaraní), Ruiz de Montoya, 1876

R\&T dialectos pano del alto Juruá, Rivet \& Tastevin, 1932

$\mathrm{SCH} \quad$ yukuna, Schauer et al., 2005

SHA nomatsiguenga, Shaver, 1996

SN matsigenka, Snell, 2011

STR nheêngatú (tupí-guaraní), Stradelli, 1929

TSC quechua, von Tschudi, 1853

TU candoshi (aislada), Tuggy, 1966

VA shiwilu (cahuapana), Valenzuela, 2013

VAL kukama-kukamiria (tupí-guaraní), Vallejos y Amías, 2015

VE orejón, Velie \& Velie, 1981

WE quechua, Weber et al., 1998 